Symptoms of depression, socioeconomic position and physical disability were adjusted for.

Results No associations were found between marital status or number of household occupants and DASH scores. Being fully retired compared to still being in main occupation was associated with a 0.73 (95\% CI: 0.16 to 1.30 ) increase in DASH points when adjusting for sex, socioeconomic position, symptoms of depressions and physical disability. When stratifying for sex, an increase in DASH points of 0.84 (95\% CI: 0.041 to 1.63$)$ was seen in females but not males fully retired.

Conclusion Results so far from this study suggest that being retired at 60-64 years, compared to still being in main employment, may be associated with improved diet quality, especially in females.

Next analyses will consider other social health exposures, including functional aspects of social relationships, and other indices of dietary intake.

\section{P19 THE MODIFYING EFFECTS OF OBESITY ON THE ASSOCIATION BETWEEN AIR POLLUTION AND STROKE; A SYSTEMATIC REVIEW}

${ }^{1,2} \mathrm{CB}$ Dillon*, ${ }^{1} \mathrm{~A}$ Callanan, ${ }^{3} \mathrm{~S}$ Hellebust, ${ }^{1} \mathrm{CM}$ Buckley, ${ }^{1,4}{ }^{4}$ O'Reilly. ${ }^{1}$ School of Public Health, University College Cork, Cork, Ireland; ${ }^{2}$ Environmental Research Institute, University College Cork, Cork, Ireland; ${ }^{3}$ School of Chemistry, University College Cork, Cork, Ireland; ${ }^{4}$ TH Chan School of Public Health, Harvard University, Cambridge, Massachusetts, USA

\subsection{6/jech-2020-SSMabstracts.115}

Background The impact of ambient air pollution on stroke is well-documented. However, the modifying effect of obesity on the association is unclear. Recent research has proposed that obese individuals are more susceptible to the effects of air pollution. The objective is to systematically screen, appraise and synthesise the evidence examining modifying effects of obesity status on the association between air pollution and stroke.

Methods Databases searched include Scopus, PubMed and Web of Science. All empirical studies published in English between January 1st 1990 - March 30th 2019 were included for review. Data items were extracted using a standardised data extraction table. A narrative synthesis of the study results was completed. Included studies were quality assessed using the Joanna Briggs Institute and modified case-crossover appraisal tools. All evidence sourced were graded according to the Scottish Intercollegiate Guidelines Network (SIGN) level of evidence criteria.

Results Of 668 titles were identified, 218 had their full-text reviewed and seven met eligibility criteria. Five cohort studies (including a nest case-crossover), one cross-sectional study and one ecological study were included in the review. Findings across studies were limited. In summary, three studies found some evidence consistent with obesity worsening the association between air pollution and stroke. Inconsistencies in exposure used and measurement, outcome assessment, and data linkage methodology existed across studies. While the overall level of evidence assigned by the SIGN criteria was good, and the review included mainly high quality cohort studies with a low risk of confounding or bias, misclassification of exposure may be present.

Conclusion Some evidence exists to suggest modifying effects of obesity on the association between air pollution and stroke. However, evidence is very weak and hampered by different study designs and outcome assessments. Thus, further research using large, nationally representative studies with stringent outcomes and exposure measurement methods in addition to fixed linking methodology between air-pollution and health data are needed while continuing the appropriate adjustment for confounding factors.

\section{P20 A SYSTEMATIC REVIEW OF THE BURDEN OF HYPERTENSION, ACCESS TO SERVICES AND PATIENT VIEWS OF HYPERTENSION IN HUMANITARIAN CRISIS SETTINGS}

${ }^{1} \mathrm{~F}$ Kidy*, 'J Keasley, 'S Shantikumar, 'W Proto, ${ }^{1} \mathrm{M}$ McGranahan, ${ }^{2} \mathrm{~A}$ Sabouni, ${ }^{1} \mathrm{O}$ Oyebode. ${ }^{1}$ Warwick Medical School, University of Warwick, Coventry, UK; ${ }^{2}$ Department of Health Sciences, University of York, York, UK

10.1136/jech-2020-SSMabstracts.116

Background Globally, the number of people affected by humanitarian crises, relating to both conflicts and natural disasters, remains at record levels. Many crisis affected populations live in settings where the epidemiological transition is underway. Even now, ischaemic heart disease and stroke combined contribute a similar proportion of deaths as conflict and terrorism in Syria, 33.83\% and 36.13\% respectively. Following the UN high level meeting on NCDs and the global commitment to Universal Health Coverage (UHC), there is increasing effort being invested in developing guidelines and processes for the management of NCDs, especially hypertension in humanitarian settings.

The objective of this study was to contribute to the discussion by answering the following points:

1. Prevalence and incidence of hypertension in populations directly affected by conflict or natural disasters.

2. Proportion diagnosed with hypertension who are aware of the diagnosis, are receiving treatment, and have achieved control.

3. Proportion with hypertension who sought treatment but did not receive it.

4. Patient knowledge of and attitude to hypertension.

Methods A literature search was carried out in five databases, looking for peer reviewed publications published since 1999. Grey literature was also searched using Google and non-governmental organisations' web pages. The population of interest was non-pregnant, non-military adults who were directly exposed to a crisis since 1999. All study types were included. Eligibility assessment, data extraction and quality appraisal were carried out in duplicate.

Results After deduplication, 11703 abstracts were screened resulting in 402 papers for full-text review. Sixty-three studies were included in the narrative synthesis. The studies reported on a wide range of crises including the wars in Syria and Iraq, the Great East Japan Earthquake, Hurricane Katrina and Palestinian refugees in the Middle East. There were few studies from Africa or central Asia. The studies predominantly assessed prevalence of hypertension and this varied with geography and age of the population. Access to care, patient understanding and patient views on hypertension were poorly examined. Most of the studies had a high risk of bias due to methods used in the diagnosis of hypertension and in the selection of representative populations. 
Discussion Hypertension is seen in all humanitarian settings and the burden can be considerable. Further studies are needed to accurately estimate prevalence of hypertension in crisis affected populations throughout the world. An appreciation of patient knowledge and understanding of hypertension as well as the cascade of care would be invaluable in informing service provision.

\section{P21 UNIVERSAL HEALTH COVERAGE IN SUB-SAHARAN AFRICA: IMPLICATIONS FOR PLANETARY HEALTH}

${ }^{1} \mathrm{SC}$ Ifeagwu*, 'J Yang, ${ }^{1,2} \mathrm{R}$ Parkes-Ratanshi, ${ }^{1} \mathrm{C}$ Brayne. 'Department of Public Health and Primary Care, University of Cambridge, Cambridge, UK; ${ }^{2}$ Infectious Diseases Institute, Makerere University College of Health Sciences, Kampala, Uganda

\subsection{6/jech-2020-SSMabstracts. 117}

Background Determinants of health in a population are related to numerous factors beyond health services. However, means to provide access to and the actual shape of health services are important for planetary health. Ability to benefit from these services is essential in order to foster sustainable development. Universal health coverage (UHC), which is embedded within the United Nations Sustainable Development Goals, is defined by the World Health Organization as all individuals and communities having access to any health services they need, of sufficient quality to be effective, without suffering financial hardship. Effective strategies for financing healthcare are critical in achieving this goal yet remain a challenge in Sub-Saharan Africa (SSA). In this respect, the aim of this review is to determine the extent of research in the published literature that examine health financing approaches and strategies for UHC in SSA.

Methods A systematic literature review was conducted in line with the Preferred Reporting Items for Systematic Reviews and Meta-Analyses (PRISMA) reporting guidelines. On 19 July 2019, MEDLINE, EMBASE, Web of Science, Global Health Database, the Cochrane Library, Scopus and JSTOR were searched for literature published from 2005. Studies that described health financing approaches and strategies for UHC in SSA were eligible for inclusion. For selected papers, reference lists were searched through the snowballing procedure for further studies. The systematic review protocol was registered with the International Prospective Register of Systematic Reviews (PROSPERO) on 14 August 2019 (registration number CRD42019142895).

Results Of the records screened, 39 papers were selected for inclusion and analysis. The results indicate that a majority of health care revenue in SSA is from direct out-of-pocket payments. Another common health financing mechanism throughout the region was donor funding, which was reported by most of the studies. Overall, many countries are starting to develop national health insurance schemes, while others have structures in place despite low population coverage of these schemes. On average, the quality score of all studies combined was $80.4 \%$, indicating a high appraisal score across the selected studies.

Discussion Appropriate health financing strategies underpin sustainable health services and the attainment of UHC is inherently linked to planetary health. It is evident from the systematic review that existing health financing strategies in SSA are inadequate and innovative solutions are needed. Thus, establishing evidence-based, multi-sectoral strategies and sustainable solutions tailored to country contexts remains imperative.

\section{P22 'YOU CANNOT BUILD A GIANT UMBRELLA OVER TUMBES WHEN EL NIÑO COMES': A QUALITATIVE STUDY OF THE PERCEPTIONS OF EL NIÑO'S IMPACT ON MENTAL HEALTH, BARRIERS TO RESILIENCE AND ACCESS TO SUPPORT IN TUMBES, PERU'}

${ }^{1,2}$ ECF Flores*, ${ }^{1}$ DF Fuhr, ${ }^{1}$ VS Simms, ${ }^{2}$ AGL Lescano, ${ }^{1} \mathrm{NT}$ Thorogood. ${ }^{1}$ Population Health, London School of Hygiene and Tropical Medicine, London, UK; ${ }^{2}$ Public Health, Universidad Peruana Cayetano Heredia, Lima, Peru

\subsection{6/jech-2020-SSMabstracts. 118}

Background Despite the well-known widespread effects on health of the El Niño phenomenon, there is little evidence of its psychosocial impact on historically affected communities and the ways that they respond to this cyclic environmental event. We aimed to describe the perceived effects on residents' mental health, and the barriers to achieving psychological wellbeing and accessing support, in a post-El Niño scenario in Tumbes, Peru, a historically high-risk area for floods and heavy rains.

Methods Between May and June 2017 we conducted 27 semistructured in-depth interviews and 3 focus group discussions with 24 adult residents of Tumbes, Peru. The participants were classified into the following subgroups: a. Representatives of the local authority based in Tumbes city; b. Representatives of the local authority based outside of Tumbes city, in the Tumbes region; c. Residents from heavily affected localities; and d. Residents from low-affected localities.

Results During early parts of the interview', the overall impact of El Niño effects on mental health was minimized. Authorities and some residents suggested that they were 'accustomed' to them. Most of the responses changed later, when they described flood-related suffering either directly experienced or witnessed. Related socioeconomic struggles were described, as well as feelings of lack of support, unfairness and helplessness. The local representatives acknowledged that effective support and aid was sometimes unavailable. Contextual factors related to corruption, underfunding of prevention activities, and lack of reconstruction programs, led to despair and distrust of authorities. Many participants allocated responsibility to specific actors, but suggested solutions or acknowledgement of personal responsibility were less commonly reported. Neither psychosocial programmes, availability of psychological therapies or community-based support programs were routinely reported. Mutual collaboration with neighbours, local authorities and aid organizations were highlighted as potentially useful, but currently non-existent as an organized or evenly distributed force. Findings suggest that psychosocial distress was exacerbated by poverty, scarcity of work, and membership of an already vulnerable group.

Conclusion The findings show the multiple and interrelated issues associated with under-resourced communities in a postdisaster context, and how socioeconomic distress and lack of support contribute to psychosocial suffering in this population. In addition to providing emergency aid and health support, these vulnerable communities would benefit from an integrated care and a resilience-promoting approach, that considers contextual and community-level distress alongside improved access to prevention measures and reconstruction activities. 